\title{
Time-Progress Evaluation for Dense-Time Automata with Concave Path Conditions ${ }^{\star}$
}

\author{
Farn Wang \\ Dept. of Electrical Engineering \\ Graduate Institute of Electronic Engineering \\ National Taiwan University \\ farn@cc.ee.ntu.edu.tw; http://cc.ee.ntu.edu.tw/ farn
}

RED 7.0 is available at http://sourceforge.net/projects/redlib

\begin{abstract}
The evaluation of successor or predecessor state spaces through time progress is a central component in the model-checking algorithm of dense-time automata. The definition of the time progress operator takes into consideration of the path condition of time progress and usually results in high complexity in the evaluation. Previous algorithms in this aspect usually assume that the original location invariance conditions of an automaton are convex in the dense-time state space. Based on this assumption, efficient algorithms for convex path conditions can be designed for reachability analysis. However, it is not clear whether the path conditions are still convex in the general setting of TCTL model-checking. In this work, we discuss the concept of time-convexity that allows us to relax the restrictions on the application of time-progress evaluation algorithm for convex path conditions. Then we give examples in TCTL model-checking that engenders time-concave path conditions even when the original automaton location invariance conditions are time-convex. Then we present two techniques that allow us to apply the evaluation algorithms for time-convex path conditions to time-concave path conditions. Finally, we report our experiment with the techniques. For some benchmarks, our techniques may enhance the performance of modelchecking by an order of magnitude.
\end{abstract}

Keywords: timed automaton, time progress, model-checking, TCTL, convex, concave

\section{Introduction}

In the last two decades, we have witnessed significant progress in both theory and applications of the model-checking technology of dense-time systems $[1,4,8,11]$. One popular framework in this regard is called TCTL model-checking [1] which assumes a given dense-time system description as a timed automaton (TA) [3] and

* The work is partially supported by NSC, Taiwan, ROC under grants NSC 95-2221E-002-067 and NSC 95-2221-E-002-072. 
a given specification formula in Timed Computation Tree Logic (TCTL) [1] and checks whether the TA satisfies the TCTL formula. The TCTL model-checking technology could be an attractive choice to the industry as long as the performance of the related verification algorithms could handle industrial projects. However, at this moment, many algorithms used in TCTL model-checking still suffer from low performance. To achieve the promise of TCTL model-checking, the performance of related algorithms has to be enhanced.

One important algorithm in TCTL model-checking is the time-progress evaluation algorithm. For simplicity, we focus on the backward time-progress operation. However, the ideas discussed in this work should also apply to the forward counterpart. Usually we are given a path condition $\phi$ and a destination condition $\psi$ and want to compute the condition, $\operatorname{Tbck}(\phi, \psi)$ in symbols, of those states that can go to a state satisfying $\psi$ through a time progression along which all states satisfying $\phi$. For convenience, given $t \in \mathbb{R}^{\geq 0}$, we let $\phi+t$ be the condition for states that satsify $\phi$ after the progression of $t$ time units [1]. Then $\operatorname{Tbck}(\phi, \psi)$ can be formulated as follows [7].

$$
\begin{aligned}
\operatorname{Tbck}(\phi, \psi) & \stackrel{\text { def }}{=} \exists t \in \mathbb{R} \geq 0\left(\psi+t \wedge \forall t^{\prime} \in \mathbb{R}^{\geq 0}\left(t^{\prime} \leq t \rightarrow \phi+t^{\prime}\right)\right) \\
& \equiv \exists t \in \mathbb{R}^{\geq 0}\left(\psi+t \wedge \neg \exists t^{\prime} \in \mathbb{R}^{\geq 0}\left(t^{\prime} \leq t \wedge \neg \phi+t^{\prime}\right)\right)
\end{aligned}
$$

The outer quantification on $t$ specifies the "through a time progression" part. The inner quantification specifies that every state along the finite computation also satisfies $\phi$. As can be seen, $\operatorname{Tbck}(\phi, \psi)$ incurs two existential quantifications (or Fourier-Motzkin elimination [6]), two complementations, and two conjunctions. Since the time-progress algorithm is fundamental to TCTL model-checking, such an involved formulation usually results in significant performance degradation.

One way to enhance the evaluation efficiency of Tbck() is to make an assumption of the TAs. An observation is that if the path condition $\phi$ characterizes a convex ${ }^{1}$ state space, then $\operatorname{Tbck}(\phi, \psi)$ can be rewritten as follows.

$$
\operatorname{Tbck}^{\prime}(\phi, \psi) \stackrel{\text { def }}{=} \exists t \in \mathbb{R} \geq 0(\psi+t \wedge \phi \wedge \phi+t)
$$

The reason is that for two states $\nu$ and $\nu^{\prime}$, that respectively represent the starting state and the destination state of a time progression, we know that the following two conditions are true.

- Both $\nu$ and $\nu^{\prime}$ are in the convex space characterized by $\phi$.

- All states that happen during this time progress actually form a straight line segment between $\nu$ and $\nu^{\prime}$.

According to the definition of convexity, then all states in this straight line segment (and time progression) must also be in the space characterized by $\phi$. As can be seen from $\operatorname{Tbck}^{\prime}()$, one existential quantification and two complementations can be avoided with this assumption. It will be interesting to see to what extent in TCTL model-checking $[1,10]$, we can use Tbck' () in place of Tbck(). According

\footnotetext{
${ }^{1}$ A space is convex if for any two points in the space, any point in the straight line segment between the two points is also in the space. A space that is not convex is concave.
} 
to our knowledge, there is no related work in this regard. In this work, we have the following contributions.

- We propose the idea of time-convexity to relax the applicability of $\mathrm{Tbck}^{\prime}()$ to concave path conditions. ${ }^{2}$

- We show that if the location invariance conditions of a TA are all timeconvex, then all path conditions used in the reachability analysis are also time-convex.

- We show that there are examples in TCTL model-checking [1] that entail the computation of time progress through time-concave path conditions even when all the location invariance conditions of the TA are time-convex.

- We present two techniques that allow us to apply $\operatorname{Tbck}^{\prime}()$ for the time progress evaluation through time-concave path conditions. For several benchmarks, the techniques have significantly enhanced the performance of our TCTL model-checker.

We have the following presentation plan. Section 2 reviews the background theory. Section 3 explains the concept of time-convexity. Section 4 investigates the possibilities of time-concave and time-convex path conditions in reachability analysis and model-checking. Sections 5 and 6 respectively present a technique for efficient time progress evaluation with time-concave path conditions. Section 7 reports our implementation and experiment. Section 8 is the conclusion.

\section{TCTL model-checking problem}

\section{$2.1 \quad$ Timed automata}

Let $\mathbb{N}$ be the set of non-negative integers, $\mathbb{Z}$ the set of all integers, and $\mathbb{R} \geq 0$ the set of non-negative reals. Also 'iff' means "if and only if." Given a set $Q$ of atomic propositions and a set $X$ of clocks, a location predicate is a Boolean combination of atoms of the forms $q$ and $x \sim c$, where $q \in Q, x \in X$, ' $\sim$ ' is one of $\leq,<,=,>, \geq$, and $c \in \mathbb{N}$. The set of all location predicates of $Q$ and $X$ is denoted as $\mathcal{L}(Q, X)$.

Definition 1. Timed automaton (TA) A $T A$ is a tuple

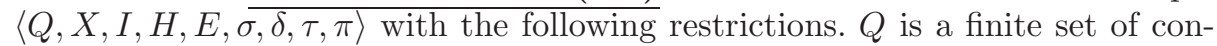
trol locations. $X$ is a finite set of clocks. $I \in \mathcal{L}(Q, X)$ is the initial condition. $H \in \mathcal{L}(Q, X)$ is the location invariance condition. $E \subseteq Q \times Q$ is a finite set of transition rules. $\sigma: E \mapsto Q$ and $\delta: E \mapsto Q$ respectively specify the source and the destination locations of each transition. $\tau: E \mapsto \mathcal{L}(\emptyset, X)$ defines the triggering condition of each rule execution. For each $e \in E, \pi(e) \subseteq X$ specifies the set of clocks to reset during the transition.

For convenience, given a TA $A=\langle Q, X, I, H, E, \sigma, \delta, \tau, \pi\rangle$, we use $Q_{A}, X_{A}, I_{A}$, $H_{A}, E_{A}, \sigma_{A}, \delta_{A}, \tau_{A}$, and $\pi_{A}$ to denote $Q, X, I, H, E, \sigma, \delta, \tau$, and $\pi$ respectively.

Example 1. We have the transition diagrams of an example TA $A$ in figure 1. The ovals represent control locations $q_{0}, q_{1}$, and $q_{2}$. Location $q_{0}$ is the initial one.

${ }^{2}$ For convenience, we say a condition is convex iff the state space that it characterizes is convex. A non-convex condition is concave. 


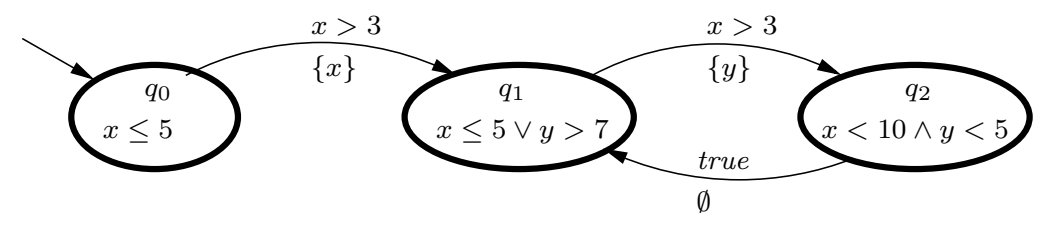

Fig. 1. An example TA

In each control location, we label the name and the constraint at that location. Thus the initial condition is $I_{A} \equiv q_{0} \wedge x \leq 5$ and the location invariance condition is $H_{A} \equiv\left(q_{0} \wedge x \leq 5\right) \vee\left(q_{1} \wedge(x \leq 5 \vee y>7)\right) \vee\left(q_{2} \wedge x<10 \wedge y<5\right)$. The arcs represent transitions between locations. On each arc, we label the triggering condition and the clock reset set.

A valuation of a set $Y$ (domain) is a mapping from $Y$ to a codomain.

Definition 2. States of a TA A clock valuation of a TA $A$ is a total valuation from $X_{A}$ to $\mathbb{R}^{\geq 0}$. A state of $A$ is a pair $(q, \nu)$ such that $q \in Q_{A}$ and $\nu$ is a clock valuation. Let $V_{A}$ denote the set of states of $A$.

For any clock valuation $\nu$ of a TA $A$ and $t \in \mathbb{R}^{\geq 0}, \nu+t$ is a valuation identical to $\nu$ except that for every $x \in X_{A},(\nu+t)(x)=\nu(x)+t$. Given a set $X^{\prime} \subseteq X_{A}$, we let $\nu X^{\prime}$ be a valuation that is identical to $\nu$ except that all variables in $X^{\prime}$ are mapped to zero.

A state $(q, \nu)$ satisfies a location predicate $\eta$, in symbols $(q, \nu) \models \eta$, if $\eta$ is evaluated true when $q$ is interpreted true, all other location names are interpreted false, and all clock variables are interpreted according to $\nu$. Given two states $(q, \nu),\left(q^{\prime}, \nu^{\prime}\right)$ and a transition $e$ of a TA $A$, we say $A$ transits with $e$ from $(q, \nu)$ to $\left(q^{\prime}, \nu^{\prime}\right)$, in symbols $(q, \nu) \stackrel{e}{\longrightarrow}\left(q^{\prime}, \nu^{\prime}\right)$, if $\sigma_{A}(e)=q, \delta_{A}(e)=q^{\prime},(q, \nu)=$ $\tau_{A}(e) \wedge H_{A}, \nu \pi_{A}(e)=\nu^{\prime}$, and $\left(q^{\prime}, \nu^{\prime}\right)=H_{A}$.

Definition 3. Runs Given a TA $A$, a run of $A$ is an infinite sequence of statetime pairs $\left(\left(q_{0}, \nu_{0}\right), t_{0}\right)\left(\left(q_{1}, \nu_{1}\right), t_{1}\right) \ldots\left(\left(q_{k}, \nu_{k}\right), t_{k}\right) \ldots \ldots$ with the following three restrictions. (1) $t_{0} t_{1} \ldots t_{k} \ldots \ldots$ is a monotonically, non-decreasing, and divergent real-number sequence. That is, $\forall k \geq 0\left(t_{k+1} \geq t_{k}\right)$ and $\forall c \in \mathbb{N} \exists k>1\left(t_{k}>c\right)$. (2) For all $k \geq 0$, for all $t \in\left[0, t_{k+1}-t_{k}\right],\left(q_{k}, \nu_{k}+t\right) \models H_{A}$. (3) For all $k \geq 0$, either $\left(q_{k}, \nu_{k}+t_{k+1}-t_{k}\right)=\left(q_{k+1}, \nu_{k+1}\right)$ or there is an $e \in E_{A}$ such that $\left(q_{k}, \nu_{k}+t_{k+1}-t_{k}\right) \stackrel{e}{\longrightarrow}\left(q_{k+1}, \nu_{k+1}\right)$. The run is initial if $\left(q_{0}, \nu_{0}\right) \models I_{A}$.

\subsection{Timed Computation Tree Logic (TCTL)}

Given a set $Q$ of atomic propositions, a set $X$ of clocks, and a $b \in \mathbb{N}$, a zone predicate within bound $b$ is a Boolean combination of atoms of the forms $q$ and $x-y \sim c$, where $q \in Q, x, y \in X \cup\{0\}, ' \sim^{\prime} \in\{<, \leq,=, \neq, \geq,>\}$, and $c \in \mathbb{Z} \cap[-b, b]$. The set of all zone predicates of $Q$ and $X$ within bound $b$ is denoted as $\mathcal{Z}_{b}(Q, X)$. The satisfaction of zone predicates by a state is defined similarly as that of location predicates. 
$T C T L$ is a language for the specification of timing behaviors with branching structure [1]. A TCTL formula $\phi$ is of the following syntax.

$$
\phi::=\eta\left|\phi_{1} \vee \phi_{2}\right| \neg \phi_{1}\left|\exists \square_{\sim_{c}} \phi_{1}\right| \exists \phi_{1} \mathcal{U}_{\sim_{c}} \phi_{2}
$$

Here $\eta$ is a zone predicate in $\mathcal{Z}_{\infty}(Q, X)^{3}, \sim \in\{<, \leq,=, \neq, \geq,>\}$, and $c$ is a non-negative integer constant. For modal formulas $\exists \square_{\sim_{c}} \phi_{1}$ and $\exists \phi_{1} \mathcal{U}_{\sim_{c}} \phi_{2}, \phi_{1}$ is called the path condition while $\phi_{2}$ is called the destination condition. Standard shorthands like true, false, $\left.\phi_{1} \wedge \phi_{2}, \phi_{1} \rightarrow \phi_{2}, \exists\right\rangle_{\sim_{c}} \phi_{1}, \forall \square_{\sim_{c}} \phi_{1}, \forall \nabla_{\sim_{c}} \phi_{1}$, and $\forall \phi_{1} \mathcal{U}_{\sim c} \phi_{2}$ are also adopted.

Note that, unlike the original definition of TCTL [1], we allow inequalities in $\mathcal{Z}_{\infty}(Q, X)$ to appear in TCTL formulas. The reason is that in the evaluation of nested modal formulas, the evaluation of inner modal formulas may yield predicates in $\mathcal{Z}_{\infty}(Q, X)$ anyway. Thus, in the general context of TCTL modelchecking, it makes no difference to have zone predicates in TCTL formulas.

Given a state $(q, \nu)$ of a TA $A$ and a TCTL formula $\phi$, we use the notation $A,(q, \nu) \models \phi$ to mean that state $(q, \nu)$ satisfies $\phi$ in $A$. The definition of satisfaction of zone (location) predicates and Boolean formulas are straightforward. Those of satisfaction of the modal formulas are as follows.

- $A,(q, \nu) \models \exists \square_{\sim_{c}} \phi_{1}$ iff there is a run from $(q, \nu)$ such that for all states $\left(q^{\prime}, \nu^{\prime}\right)$ that is $t$ time units from $(q, \nu)$ in the run with $t \sim c, A,\left(q^{\prime}, \nu^{\prime}\right) \models \phi_{1}$.

- $A,(q, \nu) \models \exists \phi_{1} \mathcal{U}_{\sim_{c}} \phi_{2}$ iff there is a run from $(q, \nu)$ such that

- there is a state $\left(q^{\prime}, \nu^{\prime}\right)$ that is $t$ time units from $(q, \nu)$ in the run with $t \sim c$ and $A,\left(q^{\prime}, \nu^{\prime}\right) \models \phi_{2}$; and

- for all states $\left(q^{\prime \prime}, \nu^{\prime \prime}\right)$ before $\left(q^{\prime}, \nu^{\prime}\right)$ in the run, $A,\left(q^{\prime \prime}, \nu^{\prime \prime}\right) \models \phi_{1}$.

The TCTL model-checking problem asks if all initial states of a TA satisfy a TCTL formula in the TA. Given a TA $A$ and a TCTL formula $\phi$, we use $\llbracket \phi \rrbracket_{A}$ to denote the state space characterized by $\phi$ in $A$. It is easy to see that for any state $(q, \nu),(q, \nu) \in \llbracket \phi \rrbracket_{A}$ iff $A,(q, \nu) \models \phi$.

For convenience, given a condition $\eta$ for a set of destination state and a transition $e$, we let $\operatorname{Xbck}_{e}(\eta)$ be the condition for states that can directly go to states in $\eta$ through transition $e$. Formally speaking, $(q, \nu) \models \operatorname{Xbck}_{e}(\eta)$ iff there exists a $\left(q^{\prime}, \nu^{\prime}\right) \models \eta$ and $(q, \nu) \stackrel{e}{\longrightarrow}\left(q^{\prime}, \nu^{\prime}\right)$. According to $[7,10]$, the formulation for the evaluation of a formula like $\exists \square_{\sim_{c}} \phi_{1}$ can be represented as follows.

$$
\exists z\left(z=0 \wedge \operatorname{gfp} Z\left(\bigvee_{e \in E_{A}} \operatorname{Tbck}\left(z \sim c \rightarrow\left(\phi_{1} \wedge H_{A}\right), \operatorname{Xbck}_{e}(Z)\right)\right)\right)
$$

Here gfp is the greatest fixpoint operator. The evaluation of the greatest fixpoint operator works by iteratively eliminating states from $Z$ until we find that there is no more elimination possible. $z$ is an auxiliary clock variable not used in $\phi_{1}$, $I_{A}, H_{A}$, and the transitions of $A$. As can be seen, formula $z \sim c \rightarrow\left(\phi_{1} \wedge H_{A}\right)$ appears in formula ( $\mathrm{T}$ ) as a path condition.

Also, the formulation for the evaluation of a formula like $\exists \phi_{1} \mathcal{U}_{\sim_{c}} \phi_{2}$ can be represented as follows.

$$
\exists z\left(z=0 \wedge \operatorname{lfp} Z .\left(\left(\phi_{2} \wedge z \sim c \wedge H_{A}\right) \vee \bigvee_{e \in E} \operatorname{Tbck}\left(\phi_{1} \wedge H_{A}, \operatorname{Xbck}_{e}(Z)\right)\right)\right)
$$

\footnotetext{
${ }^{3}$ We abuse the notation $[-\infty, \infty]$ for $(-\infty, \infty)$.
} 
Here lfp is the least fixpoint operator. It characterizes the space of those states that can reach states satisfying $\phi_{2}$ through a run segment along which all states satisfy $\phi_{1}$. The evaluation of the least fixpoint operator works by iteratively adding states to $Z$ until we find that there is no more addition possible. $z$ is an auxiliary clock variable not used in $\phi_{1}, \phi_{2}, I_{A}, H_{A}$, and the transitions of $A$. As can be seen, formula $\phi_{1} \wedge H_{A}$ appears in formula (T) as a path condition.

\section{Zones, convexity, and time-convexity}

Given a TA $A$ and a TCTL formula $\phi$, we let $C_{A}^{\phi}$ be the biggest timing constant used in $A$ and $\phi$. A clock zone of $A$ and $\phi$ is a set of clock valuations characterizable with a conjunctive ${ }^{4}$ zone-predicate in $\mathcal{Z}_{C_{A}^{\phi}}\left(\emptyset, X_{A}\right)$. A clock zone is a convex space of clock valuations. Without loss of generality, we assume that the given characterization zone predicate for a clock zone is always tight. That is, for every inequality $x-y \sim c$ in the characterization zone predicate, we cannot change the value of $c$ without changing the members of the corresponding clock zone. Such a tight zone predicate for a clock zone can be obtained with an all-pair shortest-path algorithm with cubic time complexity [5].

A zone of $A$ and $\phi$ is a set of states in $V_{A}$ characterizable with a conjunctive zone-predicate like $q \wedge \eta$ with a $q \in Q_{A}$ and $\eta \in \mathcal{Z}_{C_{A}^{\phi}}\left(\emptyset, X_{A}\right)$. The states in a zone share the same control location. According to [7], the state spaces of $A$ that we need to manipulate in model-checking for $\phi$ are finite unions of zones. Such a union can be characterized with zone predicates in $\mathcal{Z}_{C_{A}^{\phi}}\left(Q_{A}, X_{A} \cup\{z\}\right)$ where $z$ is an auxiliary clock variable not used in $A[7,10]$. Many model-checkers for TAs are based on symbolic manipulation algorithms of zone predicates represented in various forms $[4,8,11]$.

For convenience, we may also represent a zone as a pair like $(q, \eta)$ with $q \in Q_{A}$ and $\eta \in \mathcal{Z}_{C_{A}^{\phi}}\left(\emptyset, X_{A} \cup\{z\}\right)$. A set $S$ of zones is convex if for each $q \in Q_{A}$, $\bigcup_{(q, \eta) \in S} \llbracket \eta \rrbracket_{A}$ is convex. If $S$ is not convex, it is concave. The reachable state space of a TA is usually concave. Most state-spaces that we need to manipulate in TCTL model-checking are likely concave. For convenience, we say a formula $\phi$ is convex iff $\llbracket \phi \rrbracket_{A}$ is convex. If $\phi$ is not convex, then it is concave.

Example 2. The initial condition $I_{A}$ of the TA in example 1 is convex while the location invariance condition $H_{A}$ is concave. Specifically, the following subformula $\dot{H} \equiv q_{1} \wedge(x \leq 5 \vee y>7)$ is concave. For example, we may have two states $\left(q_{1}, \nu_{1}\right)$ and $\left(q_{1}, \nu_{2}\right)$ with $\nu_{1}(x)=\nu_{1}(y)=4$ and $\nu_{2}(x)=\nu_{2}(y)=8$. It is clear that $\left(q_{1}, \nu_{1}\right) \in \llbracket \dot{H} \rrbracket_{A}$ and $\left(q_{1}, \nu_{2}\right) \in \llbracket \dot{H} \rrbracket_{A}$. However, the middle point, say $\left(q_{1}, \nu_{3 / 2}\right)$, between $\left(q_{1}, \nu_{1}\right)$ and $\left(q_{1}, \nu_{2}\right)$ with $\nu_{3 / 2}(x)=\nu_{3 / 2}(y)=6$ is not in $\llbracket \dot{H} \rrbracket_{A}$.

Concavity may also happen with difference constraints between two clocks. For example, the following zone predicate $\ddot{H} \equiv q_{1} \wedge(x-y<3 \vee x-y>7)$ is also concave. For example, we may have two states $\left(q_{1}, \nu_{3}\right)$ and $\left(q_{1}, \nu_{4}\right)$ with

\footnotetext{
${ }^{4}$ A conjunctive predicate does not have negation and disjunction in it.
} 
$\nu_{3}(x)=9, \nu_{3}(y)=1, \nu_{4}(x)=1$, and $\nu_{4}(y)=9$. It is clear that $\left(q_{1}, \nu_{3}\right)$ and $\left(q_{1}, \nu_{4}\right)$ are both in $\llbracket \ddot{H} \rrbracket_{A}$. However the middle point, say $\left(q_{1}, \nu_{7 / 2}\right)$, between $\left(q_{1}, \nu_{1}\right)$ and $\left(q_{1}, \nu_{2}\right)$ with $\nu_{7 / 2}(x)=\nu_{7 / 2}(y)=5$ is not in $\llbracket \ddot{H} \rrbracket_{A}$.

It is known that procedure $\operatorname{Tbck}^{\prime}()$ for time progress evaluation can be applied to convex path conditions.

Example 3. In example 1, $\operatorname{Tbck}^{\prime}()$ is not applicable to $H_{A}$ which is concave. For example, $\operatorname{Tbck}\left(H_{A}, q_{1} \wedge x=10 \wedge y=10\right)$ is $q_{1} \wedge x>7 \wedge y>7 \wedge x \leq 10 \wedge y \leq$ $10 \wedge x-y=0$. However, $\operatorname{Tbck}^{\prime}\left(H_{A}, q_{1} \wedge x=10 \wedge y=10\right)$ is $q_{1} \wedge x \geq 0 \wedge y \geq$ $0 \wedge x \leq 10 \wedge y \leq 10 \wedge x-y=0$ which is incorrect.

Here we relax the restriction of the applicability of $\operatorname{Tbck}^{\prime}()$ with the following concept.

Definition 4. Time-convexity A union $U$ of clock zones is time-convex iff for any $\nu \in U$ and $t \in \mathbb{R}^{\geq 0}$ with $\nu+t \in U$, then for any $t^{\prime} \in[0, t], \nu+t^{\prime} \in U$. Otherwise, it is called time-concave. A set $S$ of zones is time-convex if for each $q \in Q_{A}, \bigcup_{(q, \eta) \in S} \llbracket \eta \rrbracket_{A}$ is time-convex; it is time-concave else.

Example 4. In examples 1 and $2, I_{A}$ is time-convex while $H_{A}$ is time-concave. Moreover, zone predicate $\ddot{H} \equiv q_{1} \wedge(x-y<3 \vee x-y>7)$ is concave. But we have the following derivation for any state $(q, \nu)$ and real $t \in \mathbb{R}^{\geq 0}$.

$$
\begin{aligned}
& \left(q_{1}, \nu\right) \models q_{1} \wedge(x-y<3 \vee x-y>7) \\
& \equiv\left(q_{1}, \nu+t\right) \models q_{1} \wedge(x+t-y-t<3 \vee x+t-y-t>7) \\
& \equiv\left(q_{1}, \nu+t\right) \models q_{1} \wedge(x-y<3 \vee x-y>7)
\end{aligned}
$$

Thus it is clear that $q_{1} \wedge(x-y<3 \vee x-y>7)$ is time-convex.

Lemma 1. Given a TA A, a time-convex path zone predicate $\phi$, and a destination zone predicate $\psi, \llbracket \operatorname{Tbck}(\phi, \psi) \rrbracket_{A}=\llbracket \operatorname{Tbck}^{\prime}(\phi, \psi) \rrbracket_{A}$.

Proof : We can prove this lemma in two directions. First, we want to prove that $\operatorname{Tbck}(\phi, \psi) \subseteq \operatorname{Tbck}^{\prime}(\phi, \psi)$. Given a state $(q, \nu) \models \operatorname{Tbck}(\phi, \psi)$, we have the following derivation.

$(q, \nu) \models \operatorname{Tbck}(\phi, \psi)$

$\equiv(q, \nu)=\exists t \in \mathbb{R}^{\geq 0}\left(\psi+t \wedge \forall t^{\prime} \in \mathbb{R}^{\geq 0}\left(t^{\prime} \leq t \rightarrow \phi+t^{\prime}\right)\right)$

$\Rightarrow(q, \nu)=\exists t \in \mathbb{R}^{\geq 0}\left(\begin{array}{c}\psi+t \wedge \phi \wedge \phi+t \\ \wedge \forall t^{\prime} \in \mathbb{R}^{\geq 0}\left(t^{\prime} \leq t \rightarrow \phi+t^{\prime}\right)\end{array}\right)$, instantiating $t^{\prime}$ with $t$

$\Rightarrow(q, \nu) \models \exists t \in \mathbb{R}^{\geq 0}(\psi+t \wedge \phi \wedge \phi+t)$, restriction relaxation.

$\equiv(q, \nu) \mid=\operatorname{Tbck}^{\prime}(\phi, \psi)$, defnition

Now we prove $\operatorname{Tbck}^{\prime}(\phi, \psi) \subseteq \operatorname{Tbck}(\phi, \psi)$ with the following derivation.

$(q, \nu) \mid=\operatorname{Tbck}^{\prime}(\phi, \psi)$

$\equiv(q, \nu) \models \exists t \in \mathbb{R}^{\geq 0}(\psi+t \wedge \phi \wedge \phi+t)$

$\Rightarrow(q, \nu) \models \exists t \in \mathbb{R}^{\geq 0}\left(\begin{array}{c}\psi+t \wedge \phi \wedge \phi+t \\ \wedge \forall t^{\prime} \in \mathbb{R}^{\geq 0}\left(t^{\prime} \leq t \rightarrow \phi+t^{\prime}\right)\end{array}\right)$, since $\phi$ is time-convex

$\equiv(q, \nu) \mid \exists t \in \mathbb{R}^{\geq 0}\left(\psi+t \wedge \forall t^{\prime} \in \mathbb{R}^{\geq 0}\left(t^{\prime} \leq t \rightarrow \phi+t^{\prime}\right)\right)$, since $t \leq t \wedge 0 \leq t$

$\equiv(q, \nu) \models \operatorname{Tbck}(\phi, \psi)$, defnition 
With the proof for the two directions, we know the lemma is correct.

Lemma 1 implies that we can also apply the more efficient $\mathrm{Tbck}^{\prime}()$ to concave but time-convex path conditions.

Example 5. In example 2, $\operatorname{Tbck}^{\prime}()$ was not thought to be applicable to path zone predicate $\ddot{H}$ either. But now, $\operatorname{Tbck}\left(\ddot{H}, q_{1} \wedge x=8 \wedge y=8\right)$ and $\operatorname{Tbck}^{\prime}\left(\ddot{H}, q_{1} \wedge x=\right.$ $8 \wedge y=8)$ both evaluate to $q_{1} \wedge x \geq 0 \wedge y \geq 0 \wedge x \leq 8 \wedge y \leq 8 \wedge x-y=0$.

\section{Time-concavity and convexity in verification problems}

In this section, we show two things for TCTL model-checking. First, timeconvexity of the location invariance condition $H_{A}$ is good enough to guarantee the time-convexity of all path conditions used in the reachability analysis of $A$. Second, time-convexity of $H_{A}$ is not good enough to guarantee the timeconvexity of all path conditions in the TCTL model-checking of $A$.

\subsection{For reachability analysis}

The most used verification framework is reachability analysis. In this framework, we are given a TA $A$ and a safety predicate $\eta$ and want to check whether there is an initial run of $A$ along which some state satisfies $\neg \eta$. The system is safe iff $A$ satisfies $\forall \square \eta \equiv \neg \exists \operatorname{true} \mathcal{U} \neg \eta$. According to formula $(\exists \mathcal{U})$ in page 5 , we find that all the path conditions used in the time progress evaluation is exactly the $H_{A}$ of a TA $A$. Thus we have the following lemma.

Lemma 2. For reachability analysis of a $T A A$, if $H_{A}$ is time-convex, then $\operatorname{Tbck}^{\prime}()$ can be used in place of $\operatorname{Tbck}()$ in formula ( $\left.\exists \mathcal{U}\right)$ without affecting the result of analysis.

\subsection{For TCTL model-checking}

We have identified some generic cases in examples 6 through 10 that can cause time-concave path conditions in model-checking.

Example 6. Disjunction in the path conditions in modal formulas. We may have a formula: $\exists\left(q_{1} \wedge(x \leq 5 \vee y>7)\right) \mathcal{U} q_{2}$. Given a TA $A$ with a timeconvex $H_{A}$, according to formula $(\exists \mathcal{U})$ in page 5 , the path condition is $q_{1} \wedge(x \leq$ $5 \vee y>7) \wedge H_{A}$. As can be checked, the path condition is time-concave when $\llbracket q_{1} \wedge x \leq 5 \wedge H_{A} \rrbracket_{A} \neq \emptyset, \llbracket q_{1} \wedge y>7 \wedge H_{A} \rrbracket_{A} \neq \emptyset$, and $\llbracket q_{1} \wedge x>5 \wedge y \leq 7 \wedge H_{A} \rrbracket_{A} \neq \emptyset$.

For another example, according to formulation $(\exists \square)$ in page 5 , formula $\exists \square\left(q_{1} \wedge(x \leq 5 \vee y>7)\right)$ also incurs time-concavity in path condition.

Example 7. Complementation in the path conditions in modal formulas. We have a formula: $\forall \triangleright\left(q_{1} \rightarrow(x>5 \wedge y \leq 7)\right)$ which can be rewritten as $\neg \exists \square\left(q_{1} \wedge(x \leq 5 \vee y>7)\right)$. According to formulation $(\exists \square)$ in page 5 , the path 


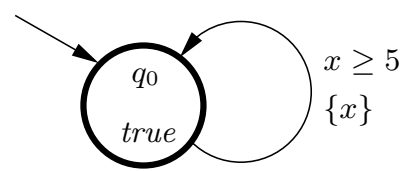

(a)

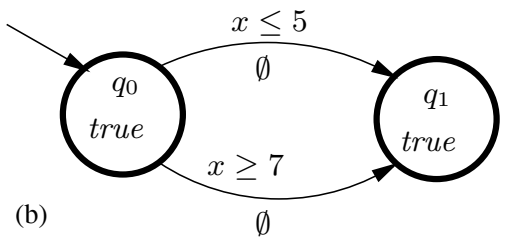

Fig. 2. Another example TA

condition $q_{1} \wedge(x \leq 5 \vee y>7) \wedge H_{A}$ is time-concave when $\llbracket q_{1} \wedge x \leq 5 \wedge H_{A} \rrbracket_{A} \neq \emptyset$, $\llbracket q_{1} \wedge y>7 \wedge H_{A} \rrbracket_{A} \neq \emptyset$, and $\llbracket q_{1} \wedge x>5 \wedge y \leq 7 \wedge H_{A} \rrbracket_{A} \neq \emptyset$.

Note that in TCTL model-checking, we usually need to calculate the complement of time-convex state spaces and end up with time-concave state spaces.

Example 8. Timing constraints with $\exists \square$-formulas. According to formula ( $\exists \square$ ) in page 5, formula: $\exists \square_{<7} x \leq 5$ incurs a path condition $z \leq 7 \rightarrow x \leq 5 \equiv$ $z>7 \vee x \leq 5$. Then following the argument in examples 2 and 4 , it is easy to see that this path condition is also time-concave.

The following two examples show that path condition concavity may also happen due to the structures of TAs.

Example 9. Time-concavities due to TA structures. We may have the example TA $A$ in figure 2(a) and want to check $A,\left(q_{0}, \nu\right) \models \exists \square \exists x>3 \mathcal{U} x \leq 0$ with $\nu(x)=0$. Note that the location invariance condition is time-convex. $\left(q_{0}, \nu\right) \in \llbracket \exists x>3 \mathcal{U} x \leq 0 \rrbracket_{A}$ since $x \leq 0$ is immediately fulfilled at $\nu$. Also $\left(q_{0}, \nu+4\right) \in \llbracket \exists x>3 \mathcal{U} x \leq 0 \rrbracket_{A}$ with the firing of the transition at $\nu+5$. But it is clear that $\left(q_{0}, \nu+1\right) \notin \llbracket \exists x>3 \mathcal{U} x \leq 0 \rrbracket_{A}$.

According to the original definition of TCTL [1], only propositions may appear as atoms. Thus we may argue that the above-mentioned formulas in examples 6 to 9 may not happen in the original TCTL definition. The following example is interesting in this regard.

Example 10. Nested $\exists \mathcal{U}$-formulas with modal timing constraints. Now we may want to check the TA in figure 2 (b) for a formula $\exists \square \exists q_{0} \mathcal{U}_{<1} q_{1}$ at a state $\left(q_{0}, \nu\right)$ with $\nu(x)=5$. Then $\left(q_{0}, \nu\right) \in \llbracket \exists q_{0} \mathcal{U}_{<1} q_{1} \rrbracket_{A}$ and $\left(q_{0}, \nu+2\right) \in \llbracket \exists q_{0} \mathcal{U}_{<1} q_{1} \rrbracket_{A}$. However, it is clear that $\left(q_{0}, \nu+1\right) \notin \llbracket \exists q_{0} \mathcal{U}_{<1} q_{1} \rrbracket_{A}$.

\section{$5 \quad$ Algorithm with cascading convexities}

We have experimented with several techniques for performance enhancement of time progress evaluation for time-concave path conditions. We present one such technique that we have found useful. The technique breaks a time-concave zone predicate into time-convex ones and then applies $\operatorname{Tbck}^{\prime}()$ on each time-convex ones for the evaluation of time progress. 
Given a zone predicate $\phi$ that describes a time-concave state space, we want to characterizes those states in $\llbracket \phi \rrbracket_{A}$ that can first go through a state outside $\llbracket \phi \rrbracket_{A}$ through time progression and then again end up at a state in $\llbracket \phi \rrbracket_{A}$. To calculate the time progress operation to such states in $\llbracket \phi \rrbracket_{A}$, we cannot use $\operatorname{Tbck}^{\prime}()$ in place of $\operatorname{Tbck}()$ since such states are evidence for the time-concavity of $\phi$. Formally speaking, such states can be characterized as follows.

$$
\text { TConcave }(\phi) \stackrel{\text { def }}{=} \phi \wedge \exists t \in \mathbb{R}^{\geq 0}\left(\phi+t \wedge \exists t^{\prime} \in \mathbb{R}^{\geq 0}\left(t^{\prime}<t \wedge(\neg \phi)+t^{\prime}\right)\right)
$$

We have the following lemma that establishes some properties of the characterizaton useful for our performance-enhancing techniques.

Lemma 3. Given a zone predicate $\phi$ for a TA $A$, $\llbracket \phi \rrbracket_{A}-\llbracket \operatorname{TConcave}(\phi) \rrbracket_{A}$ is time-convex.

Proof : This is straightforward from the definition of TConcave $(\phi)$.

Given two state spaces $S$ and $S^{\prime}$, we say $S$ is time-connected to $S^{\prime}$ if there is a state $(q, \nu) \in S$ and a $t \in \mathbb{R}^{\geq 0}$ such that $(q, \nu+t) \in S^{\prime}$ and for every $t^{\prime} \in[0, t]$, $\left(q, \nu+t^{\prime}\right) \in S \cup S^{\prime}$. If $S$ is not time-connected to $S^{\prime}$, then it is time-disconnected to $S^{\prime}$. The concept of time-connectivity is important for the correctness of piecewise evaluation of time progress.

Lemma 4. Suppose we are given two zone predicates $\phi$ and $\phi^{\prime}$ such that $\llbracket \phi \rrbracket_{A}$ is not time-connected to $\llbracket \phi^{\prime} \rrbracket_{A}$ and vice versa. Then for every zone predicate $\psi$, $\llbracket \operatorname{Tbck}(\phi, \psi) \rrbracket_{A} \cup \llbracket \operatorname{Tbck}\left(\phi^{\prime}, \psi\right) \rrbracket_{A}=\llbracket \operatorname{Tbck}\left(\phi \vee \phi^{\prime}, \psi\right) \rrbracket_{A}$.

Proof : It is easy to see that $\llbracket \operatorname{Tbck}(\phi, \psi) \rrbracket_{A} \cup \llbracket \operatorname{Tbck}\left(\phi^{\prime}, \psi\right) \rrbracket_{A} \subseteq \llbracket \operatorname{Tbck}\left(\phi \vee \phi^{\prime}, \psi\right) \rrbracket_{A}$. On the other hand, $\llbracket \operatorname{Tbck}(\phi, \psi) \rrbracket_{A} \cup \llbracket \operatorname{Tbck}\left(\phi^{\prime}, \psi\right) \rrbracket_{A} \supseteq \llbracket \operatorname{Tbck}\left(\phi \vee \phi^{\prime}, \psi\right) \rrbracket_{A}$ is false if either of the following two cases are true.

- There is a state $(q, \nu) \in \llbracket \phi \rrbracket_{A}$ and a $t \in \mathbb{R}^{\geq 0}$ such that $(q, \nu+t) \in \llbracket \phi^{\prime} \rrbracket_{A}$ and for every $t^{\prime} \in[0, t],\left(q, \nu+t^{\prime}\right) \in \llbracket \phi \rrbracket_{A} \cup \llbracket \phi^{\prime} \rrbracket_{A}$. But this exactly violates the assumption that $\llbracket \phi \rrbracket_{A}$ is not time-connected to $\llbracket \phi^{\prime} \rrbracket_{A}$.

- There is a state $(q, \nu) \in \llbracket \phi^{\prime} \rrbracket_{A}$ and a $t \in \mathbb{R}^{\geq 0}$ such that $(q, \nu+t) \in \llbracket \phi \rrbracket_{A}$ and for every $t^{\prime} \in[0, t],\left(q, \nu+t^{\prime}\right) \in \llbracket \phi \rrbracket_{A} \cup \llbracket \phi^{\prime} \rrbracket_{A}$. This is symmetric to the first case and violates the assumption that $\llbracket \phi^{\prime} \rrbracket_{A}$ is not time-connected to $\llbracket \phi \rrbracket_{A}$. Since both of these cases are false, we know the lemma is proven.

Lemma 5. Given a TA $A$ and a zone predicate $\phi, \llbracket \phi \rrbracket_{A}-\llbracket$ TConcave $(\phi) \rrbracket_{A}$ is not time-connected to $\llbracket$ TConcave $(\phi) \rrbracket_{A}$ and vice versa.

Proof : We first assume that $\llbracket \phi \rrbracket_{A}-\llbracket T$ Concave $(\phi) \rrbracket_{A}$ is time-connected to $\llbracket T \operatorname{Concave}(\phi) \rrbracket_{A}$. This implies that there is a state $(q, \nu) \in \llbracket \phi \rrbracket_{A}-\llbracket T$ Concave $(\phi) \rrbracket_{A}$ and a $t \in \mathbb{R}^{\geq 0}$ such that $(q, \nu+t) \in \llbracket T$ Concave $(\phi) \rrbracket_{A}$ and for each $t^{\prime} \in[0, t]$, $\left(q, \nu+t^{\prime}\right) \in \llbracket \phi \rrbracket_{A}$. According to the definition of TConcave $(\phi)$, there is a $t^{\prime \prime} \in \mathbb{R} \geq 0$ such that $\left(q, \nu+t+t^{\prime \prime}\right) \in \llbracket \phi \rrbracket_{A}$ while there is a $\bar{t}$ such that $t<\bar{t}<t+t^{\prime \prime}$ and $(q, \nu+$ $\bar{t}) \notin \llbracket \phi \rrbracket_{A}$. This contradicts our assumption that $(q, \nu) \in \llbracket \phi \rrbracket_{A}-\llbracket T$ Concave $(\phi) \rrbracket_{A}$.

We then assume that $\llbracket$ TConcave $(\phi) \rrbracket_{A}$ is time-connected to $\llbracket \phi \rrbracket_{A}-\llbracket T C$ Concave $(\phi) \rrbracket_{A}$. This implies that there is a state $(q, \nu) \in \llbracket T$ Concave $(\phi) \rrbracket_{A}$ and a $t \in \mathbb{R}^{\geq 0}$ such that $(q, \nu+t) \in \llbracket \phi \rrbracket_{A}-\llbracket$ TConcave $(\phi) \rrbracket_{A}$ and for each $t^{\prime} \in[0, t],\left(q, \nu+t^{\prime}\right) \in \llbracket \phi \rrbracket_{A}$. According to the definition of TConcave $(\phi)$, there 
is a $t^{\prime \prime} \in \mathbb{R}^{\geq 0}$ such that $\left(q, \nu+t^{\prime \prime}\right) \in \llbracket \phi \rrbracket_{A}$ while there is a $\bar{t}$ such that $0<\bar{t}<t^{\prime \prime}$ and $(q, \nu+\bar{t}) \notin \llbracket \phi \rrbracket_{A}$. With the assumption on $t$, we know that $0 \leq t<\bar{t}<t^{\prime \prime}$. This implies that $(q,(\nu+t)+\bar{t}-t) \notin \llbracket \phi \rrbracket_{A}$ and $\left(q,(\nu+t)+t^{\prime \prime}-t\right) \in \llbracket \phi \rrbracket_{A}$. This contradicts the assumption that $(q, \nu+t) \in \llbracket \phi \rrbracket_{A}-\llbracket$ TConcave $(\phi) \rrbracket_{A}$.

Based on lemmas 3, 4, and 5, we present the following procedure that breaks a zone predicate $\phi$ into a finite set of zone predicates such that for each two state predicates $\phi_{1}, \phi_{2}$ in the set, $\llbracket \phi_{1} \rrbracket_{A}$ is not time-connected to $\llbracket \phi_{2} \rrbracket_{A}$.

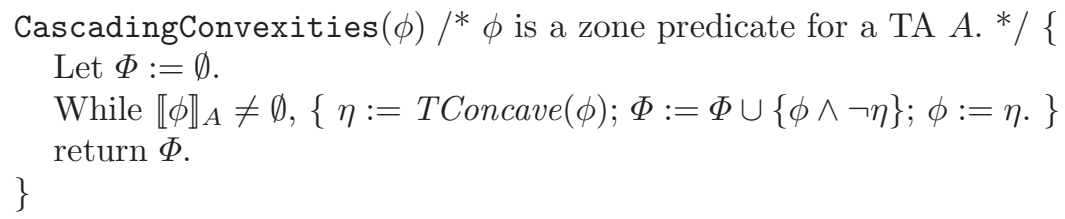

Note that this procedure terminates since the number of zone sets is finite. Thus we repeatedly remove some zones from $\llbracket \phi \rrbracket_{A}$ and eventually reduce it to $\emptyset$. According to lemma 3, we know that every zone predicate in CascadingConvexities $(\phi)$ characterizes a time-convex state space. Moreover, with lemma 3,4 , and 5 , we can establish the following lemma for a new formulation of backward time progress evaluation.

Lemma 6. For every two zone predicates $\quad \phi \quad$ and $\psi$,
$\llbracket \operatorname{Tbck}(\phi, \psi) \rrbracket_{A}=\bigcup_{\eta \in \text { CascadingConvexities }(\phi)} \operatorname{Tbck}^{\prime}(\eta, \psi)$.

\section{Algorithm with approximate time-concavity checking}

As can be seen in section 5, procedure TConcave() can be executed many times in procdeure CascadingConvexities() and incur great computation cost. We want to investigate if it may pay off to use an alternative technique that avoids the evaluation of procedure TConcave(). Given a path zone predicate $\phi$ and a destination zone predicate $\psi$, this alternative technique works in the following two steps.

(1) Partition $\phi$ into two zone predicates $\phi_{1}$ and $\phi_{2}$ such that $\llbracket \phi_{1} \rrbracket_{A} \cap \llbracket \phi_{1} \rrbracket_{A}=\emptyset$, $\llbracket \phi_{1} \rrbracket_{A} \cup \llbracket \phi_{1} \rrbracket_{A}=\llbracket \phi \rrbracket_{A}, \llbracket \phi_{1} \rrbracket_{A}$ is time-convex, and $\llbracket \phi_{1} \rrbracket_{A}$ and $\llbracket \phi_{2} \rrbracket_{A}$ are timedisconnected to each other.

(2) Then we return $\operatorname{Tbck}^{\prime}\left(\phi_{1}, \psi\right) \vee \operatorname{Tbck}\left(\phi_{2}, \psi\right)$ as the result of time progress evaluation.

The performance of the technique relies on the efficiency in carrying out step (1). We have the following lemma that helps us carrying out step (1). First, we need some notations for the convenience of discussion. Given a zone predicate $\phi$, we assume that we can construct a set $Z$ oneSet $(\phi)$ with zone elements of the form $(q, \eta)$ such that $\llbracket \phi \rrbracket_{A}=\llbracket \bigvee_{(q, \eta) \in \operatorname{ZoneSet}(\phi)} q \wedge \eta \rrbracket_{A}$. Depending on the implementation of $\phi$, there are various ways to do this. If $\phi$ is implemented with DBMs [5], then $\phi$ should already have been represented as ZoneSet $(\phi)$ with $\eta$ 
represented as a DBM for each $(q, \eta) \in \operatorname{ZoneSet}(\phi)$. If $\phi$ is implemented as a CRD (Clock-Restriction Diagram) [8], then each $(q, \eta) \in \operatorname{ZoneSet}(\phi)$ corresponds to a path in the CRD.

Given a $q \in Q$, we let $Z_{\text {oneSet }}(\phi)=\{(q, \eta) \mid(q, \eta) \in \operatorname{ZoneSet}(\phi)\}$. Also, given a set $\Phi$ of zone predicates and two clocks $x, y \in X_{A} \cup\{0\}$, we let $U B_{x-y}(\Phi)$ be the minimum upper-bound for expression $x-y$ in all states $(q, \nu) \in \mathbb{V _ { ( q , \eta ) \in \Phi }} q \wedge$ $\eta \rrbracket_{A}$. That is, $U B_{x-y}(\Phi)=\min \left\{u \mid(q, \nu) \in \llbracket \bigvee_{(q, \eta) \in \Phi} q \wedge \eta \rrbracket_{A}, \nu(x)-\nu(y) \leq u\right\} .^{5}$ If $U B_{x-y}(\Phi)$ does not exist, then we denote $U B_{x-y}(\Phi)=\infty$.

Moreover, we define a predicate ConcavityNecessary $($ () of two zone representations that share the same control location. Specifically, given two such zone representations $(q, \eta)$ and $\left(q, \eta^{\prime}\right)$, ConcavityNecessary $\left((q, \eta),\left(q, \eta^{\prime}\right)\right)$ is true if and only if there are two clocks $x, y \in X_{A} \cup\{z\}$ such that

- $U B_{x-0}(\{(q, \eta)\})<U B_{x-0}\left(\operatorname{ZoneSet}_{q}(\phi)\right)$;

- $U B_{0-y}\left(\left\{\left(q, \eta^{\prime}\right)\right\}\right)<U B_{0-y}\left(\operatorname{ZoneSet}_{q}(\phi)\right)$; and

- for all $z, w \in X_{A} \cup\{z\}, U B_{z-w}(\{(q, \eta)\})+U B_{w-z}\left(\left\{\left(q, \eta^{\prime}\right)\right\}\right) \geq 0$.

Lemma 7. Given a zone predicate $\phi$, if $\phi$ is time-concave, then there are $(q, \eta)$, $\left(q, \eta^{\prime}\right) \in$ ZoneSet $(\phi)$ satisfying ConcavityNecessary $_{\phi}\left((q, \eta),\left(q, \eta^{\prime}\right)\right)$.

Proof : We assume there is a state $(q, \nu)$ and two reals $t^{\prime}<t \in \mathbb{R}^{\geq 0}$ such that $(q, \nu),(q, \nu+t) \in \llbracket \phi \rrbracket_{A}$ while $\left(q, \nu+t^{\prime}\right) \notin \llbracket \phi \rrbracket_{A}$. Assume that there are $\left(q, \eta_{1}\right),\left(q, \eta_{2}\right) \in \operatorname{ZoneSet}(\phi)$ such that $(q, \nu) \in \llbracket q \wedge \eta_{1} \rrbracket_{A}$ and $(q, \nu+t) \in \llbracket q \wedge$ $\eta_{2} \rrbracket_{A}$. Note that for all clocks $z, w \in X_{A}, \nu(z)-\nu(w)=(\nu+t)(z)-(\nu+$ $t)(w)=\left(\nu+t^{\prime}\right)(z)-\left(\nu+t^{\prime}\right)(w)$. This implies that there is a clock $x \in X_{A}$ such that $U B_{x-0}\left(\left\{\left(q, \eta_{1}\right)\right\}\right)<\left(\nu+t^{\prime}\right)(x)<(\nu+t)(x) \leq U B_{x-0}\left(\left\{\left(q, \eta_{2}\right)\right\}\right) \leq$ $U B_{x-0}\left(\operatorname{ZoneSet}_{q}(\phi)\right)$. This implies that $U B_{x-0}\left(\left\{\left(q, \eta_{1}\right)\right\}\right)<U B_{x-0}\left(\operatorname{ZoneSet}_{q}(\phi)\right)$. This means that the first bullet is correct. Similarly, the second bullet is correct.

Note that for all clocks $z, w \in X_{A}, \nu(z)-\nu(w)=(\nu+t)(z)-(\nu+t)(w)$ which implies that $0=\nu(z)-\nu(w)+(\nu+t)(w)-(\nu+t)(z)$. According to the definition of $U B_{z-w}()$ and $U B_{w-z}()$, we know that $\nu(z)-\nu(w) \leq U B_{z-w}\left(\left\{\left(q, \eta_{1}\right)\right\}\right)$ and $(\nu+t)(w)-(\nu(z)+t) \leq U B_{w-z}\left(\left\{\left(q, \eta_{2}\right)\right\}\right)$. Thus the third bullet is also proven for $\left(q, \eta_{1}\right)$ and $\left(q, \eta_{2}\right)$.

By letting $\eta$ and $\eta^{\prime}$ be $\eta_{1}$ and $\eta_{2}$ respectively, the lemma is proven.

Based on lemma 7, for step (1) in the above, we let

$$
\phi_{2}=\bigvee_{(q, \eta),\left(q, \eta^{\prime}\right) \in \text { ZoneSet }_{(\phi), \text { ConcavityNecessary }}\left((q, \eta),\left(q, \eta^{\prime}\right)\right)}(q \wedge \eta)
$$

and $\phi_{1}=\phi \wedge \neg \phi_{2}$. We have the following lemma that shows this is indeed what we can use in step (1).

Lemma 8. With the $\phi_{1}$ and $\phi_{2}$ defined in the last paragraph, for any $\psi$, $\llbracket \operatorname{Tbck}(\phi, \psi) \rrbracket_{A}=\llbracket \operatorname{Tbck}^{\prime}\left(\phi_{1}, \psi\right) \vee \operatorname{Tbck}\left(\phi_{2}, \psi\right) \rrbracket_{A}$.

Proof : The lemma is true if $\phi_{1}$ is time-convex and $\phi_{1}, \phi_{2}$ are time-disconnected to each other. If $\phi_{1}$ is time-concave, then according to lemma 7, there are $(q, \eta),\left(q, \eta^{\prime}\right) \in$ ZoneSet $\left._{(} \phi_{1}\right)$ such that ConcavityNecessary $\phi\left((q, \eta),\left(q, \eta^{\prime}\right)\right)$ is true. Then no states in $\llbracket(q \wedge \eta) \vee\left(q \wedge \eta^{\prime}\right) \rrbracket_{A}$ should be in $\llbracket \phi_{1} \rrbracket_{A}$. This is a contradiction.

\footnotetext{
${ }^{5}$ For convenience, we let $\nu(0)=0$.
} 
If $\phi_{1}$ is time-connected to $\phi_{2}$, then there are $(q, \eta) \in \operatorname{ZoneSet}\left(\phi_{1}\right)$ and $\left(q, \eta^{\prime}\right),\left(q, \eta^{\prime \prime}\right) \in \operatorname{ZoneSet}\left(\phi_{2}\right)$ such that $\llbracket q \wedge \eta \rrbracket_{A}$ is time-connected to $\llbracket q \wedge \eta^{\prime} \rrbracket_{A}$ and ConcavityNecessary $\left(\left(q, \eta^{\prime}\right),\left(q, \eta^{\prime \prime}\right)\right) \quad$ is true. This implies ConcavityNecessary $_{\phi}\left((q, \eta),\left(q, \eta^{\prime \prime}\right)\right)$ which is also a contradiction.

With an argument similar to the one in the last paragraph, we can also prove that $\phi_{2}$ is not time-connected to $\phi_{1}$. Thus the lemma is proven.

Finally, the technique has been realized with a symbolic manipulation algorithm for zone predicates represented with CRD.

\section{Implementation and experiments}

We have implemented our ideas in sections 5 and 6 in RED 7.0, a model-checker for TAs and a parametric safety analyzer for LHAs (linear hybrid automata) [2] based on CRD and HRD (Hybrid-Restriction Diagram) technology [8,9]. We used the following two parameterized benchmarks from the literature.

1. Fischer's timed mutual exclusion algorithm [8]: The algorithm relies on a global lock and a local clock per process to control access to the critical section. Three timing constants used are 10, 19, and 30. The first formula that we check is the following.

$$
\forall \square \neg\left(\exists\left(\operatorname{critical}_{1}\right) \mathcal{U}\left(\left(\neg \operatorname{critical}_{1}\right) \wedge \exists \bigotimes_{<19} \text { critical }_{1}\right)\right)
$$

This formula intends to say that if process one leaves the critical section, then it cannot enter the critical section again in 19 time units. However, since the $\exists \mathcal{U}$-formula can be satisfied at a state that directly fulfills $\left(\neg\right.$ critical $\left.l_{1}\right) \wedge$ $\exists\rangle_{<19} \mathrm{critical}_{1}$, the formula is not satisfied.

The second formula is the following.

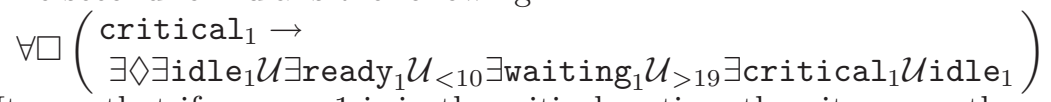

It says that if process 1 is in the critical section, then it can go through an expected mode sequence with timing restrictions back to the idle mode.

The third formula is the following.

$$
\left.\forall \square\left(\text { ready }_{1} \rightarrow \forall \diamond_{<10}\left(\text { waiting }_{1} \wedge \forall \square\left(\text { critical }_{1} \rightarrow \forall\right\rangle_{<30} \text { idle }_{1}\right)\right)\right)
$$

The formula says that if process 1 is in the ready mode, it enters the waiting mode in 10 time units and from that point on, if it enters the critical section, it returns to the idle mode in 30 time units. Note that the formula is not satisfied with Zeno computations ${ }^{6}$. So we have to use option '-Z' for quantification for non-Zeno computations.

2. CSMA/CD [11]: This is the Ethernet bus arbitration protocol with collisionand-retry. The timing constants used are 26, 52, and 808 . The first property that we want to check is the following.

$$
\forall \square\left(\left(\text { transm }_{1} \wedge x_{1}=52\right) \rightarrow \forall \square<756 \neg \text { transm }_{2}\right)
$$

It says that if sender 1 is in the transmission mode for 52 time units, then in all computations, sender 2 cannot be in the transmission mode for at least 756 time units.

The second formula is as follows.

\footnotetext{
${ }^{6}$ A Zeno computation runs forever without time converging to a finite value.
} 
Table 1. Performance data of scalability w.r.t. various strategies

\begin{tabular}{|c|c|c|c|c|c|c|c|c|c|}
\hline \multirow[b]{2}{*}{ benchmarks } & \multirow{2}{*}{$\begin{array}{l}\text { TCTL } \\
\text { spec's }\end{array}$} & \multirow[b]{2}{*}{$m$} & \multicolumn{2}{|c|}{$\operatorname{Tbck}()$} & \multicolumn{2}{|c|}{ Cascading } & \multicolumn{2}{|c|}{$A T C C$} & \multirow[b]{2}{*}{ answer } \\
\hline & & & time & mem & time & mem & time & mem & \\
\hline \multirow{9}{*}{$\begin{array}{l}\text { Fischer's } \\
\text { mutual exclusion } \\
\text { ( } m \text { processes })\end{array}$} & \multirow{3}{*}{ (C) } & 3 & $0.024 \mathrm{~s}$ & $186 \mathrm{k}$ & $0.004 \mathrm{~s}$ & $186 \mathrm{k}$ & $0.012 \mathrm{~s}$ & $3.1 \mathrm{M}$ & \multirow{3}{*}{ violated } \\
\hline & & 4 & $0.42 \mathrm{~s}$ & $32 \mathrm{M}$ & $0.044 \mathrm{~s}$ & $413 \mathrm{k}$ & $0.044 \mathrm{~s}$ & $13 \mathrm{M}$ & \\
\hline & & 5 & $8.38 \mathrm{~s}$ & $162 \mathrm{M}$ & $1.80 \mathrm{~s}$ & $69 \mathrm{M}$ & $1.83 \mathrm{~s}$ & $71 \mathrm{M}$ & \\
\hline & \multirow{3}{*}{ (D) } & 3 & $0.008 \mathrm{~s}$ & $186 \mathrm{k}$ & $0.008 \mathrm{~s}$ & $186 \mathrm{k}$ & $0.012 \mathrm{~s}$ & $2.7 \mathrm{M}$ & \multirow{3}{*}{ satisfied } \\
\hline & & 4 & $0.060 \mathrm{~s}$ & $413 \mathrm{k}$ & $0.044 \mathrm{~s}$ & $413 \mathrm{k}$ & $0.036 \mathrm{~s}$ & $9.9 \mathrm{M}$ & \\
\hline & & 5 & $4.18 \mathrm{~s}$ & $116 \mathrm{M}$ & $0.588 \mathrm{~s}$ & $34 \mathrm{M}$ & $0.536 \mathrm{~s}$ & $40 \mathrm{M}$ & \\
\hline & \multirow{3}{*}{ (E) } & 3 & $0.036 \mathrm{~s}$ & $186 \mathrm{k}$ & $0.032 \mathrm{~s}$ & $186 \mathrm{k}$ & $0.032 \mathrm{~s}$ & $6.5 \mathrm{M}$ & \multirow{3}{*}{\begin{tabular}{|l} 
satisfied \\
with \\
non-Zenoness \\
\end{tabular}} \\
\hline & & 4 & $1.06 \mathrm{~s}$ & $59 \mathrm{M}$ & $0.048 \mathrm{~s}$ & $413 \mathrm{k}$ & $0.128 \mathrm{~s}$ & $25 \mathrm{k}$ & \\
\hline & & 5 & $26.9 \mathrm{~s}$ & $344 \mathrm{M}$ & $5.36 \mathrm{~s}$ & $113 \mathrm{M}$ & $4.25 \mathrm{~s}$ & $116 \mathrm{M}$ & \\
\hline \multirow{11}{*}{$\begin{array}{l}\text { CSMA } / \mathrm{CD} \\
(1 \text { bus }+m \text { senders })\end{array}$} & \multirow{3}{*}{$(\mathrm{F})$} & 2 & $0.024 \mathrm{~s}$ & $168 \mathrm{k}$ & $0.048 \mathrm{~s}$ & $168 \mathrm{k}$ & $0.028 \mathrm{~s}$ & $2.5 \mathrm{M}$ & \multirow{3}{*}{ satisfied } \\
\hline & & 3 & $0.072 \mathrm{~s}$ & $333 \mathrm{k}$ & $0.068 \mathrm{~s}$ & $333 \mathrm{k}$ & $0.116 \mathrm{~s}$ & $20 \mathrm{M}$ & \\
\hline & & 4 & $5.49 \mathrm{~s}$ & 100M & $4.38 \mathrm{~s}$ & $90 \mathrm{M}$ & $4.72 \mathrm{~s}$ & 109M & \\
\hline & \multirow{4}{*}{ (G) } & 2 & $0.028 \mathrm{~s}$ & $168 \mathrm{k}$ & $0.028 \mathrm{~s}$ & $168 \mathrm{k}$ & $0.020 \mathrm{~s}$ & $1.2 \mathrm{M}$ & \multirow[t]{2}{*}{ violated } \\
\hline & & \begin{tabular}{|l|}
3 \\
\end{tabular} & $0.104 \mathrm{~s}$ & $333 \mathrm{k}$ & $0.092 \mathrm{~s}$ & $333 \mathrm{k}$ & $0.088 \mathrm{~s}$ & $4.1 \mathrm{M}$ & \\
\hline & & 2 & $0.096 \mathrm{~s}$ & $168 \mathrm{k}$ & $0.068 \mathrm{~s}$ & $168 \mathrm{k}$ & $0.096 \mathrm{~s}$ & $166 \mathrm{k}$ & \multirow{2}{*}{$\begin{array}{l}\text { violated with } \\
\text { non-Zenoess }\end{array}$} \\
\hline & & 3 & $108 \mathrm{~s}$ & $662 \mathrm{M}$ & $93.2 \mathrm{~s}$ & $584 \mathrm{M}$ & $96.7 \mathrm{~s}$ & $605 \mathrm{M}$ & \\
\hline & \multirow{4}{*}{$(\mathrm{H})$} & 2 & $0.040 \mathrm{~s}$ & $168 \mathrm{k}$ & $0.032 \mathrm{~s}$ & $168 \mathrm{k}$ & $0.076 \mathrm{~s}$ & $1.6 \mathrm{M}$ & \multirow[t]{2}{*}{ violated } \\
\hline & & \begin{tabular}{|l|}
3 \\
\end{tabular} & $0.100 \mathrm{~s}$ & $333 \mathrm{k}$ & $0.084 \mathrm{~s}$ & $333 \mathrm{k}$ & $0.100 \mathrm{~s}$ & $5.6 \mathrm{M}$ & \\
\hline & & \begin{tabular}{|l|}
2 \\
\end{tabular} & $0.13 \mathrm{~s}$ & $168 \mathrm{k}$ & $0.092 \mathrm{~s}$ & $168 \mathrm{k}$ & $0.152 \mathrm{~s}$ & $24 \mathrm{M}$ & \multirow{2}{*}{$\begin{array}{l}\text { satisfied with } \\
\text { non-Zenoess }\end{array}$} \\
\hline & & 3 & $266 \mathrm{~s}$ & 1199M & $214 \mathrm{~s}$ & $1036 \mathrm{M}$ & $222 \mathrm{~s}$ & $1082 \mathrm{M}$ & \\
\hline
\end{tabular}
data collected on a Pentium $41.7 \mathrm{GHz}$ with $2 \mathrm{G}$ memory running LINUX; $\mathrm{s}$ : seconds; k: kilobytes of memory in diagram data-structure; M: megabytes of memory in diagram data-structure

\section{$\forall \square \neg\left(\exists \operatorname{transm}_{1} \mathcal{U}\left(\operatorname{transm}_{2} \wedge \exists \square<26 \neg \operatorname{retry}_{1}\right)\right)$}

It intends to say that it is not possible that sender 1 remains in the transmission mode until sender 2 also does so and sender 1 does not enter the retry mode in 26 time units. This formula is not satisfied since the $\exists \mathcal{U}$-formula can be satisfied immediately with a state that directly fulfills transm t $_{2} \wedge$ $\exists \square_{<26} \neg$ retry $_{1}$. From that state on, there is a computation along which sender 2 stays in the transmission mode for 808 time units.

The final formula is the following.

$$
\left.\left.\forall \square \text { (bus_collision } \rightarrow \forall\left(\forall \diamond_{<52} \text { (wait }_{1} \vee \text { retry }_{1}\right)\right) \mathcal{U}_{<52} \text { bus_idle }\right)
$$

Note that the formula is not satisfied with Zeno computations. So we have to use option '-Z' for quantification for non-Zeno computations.

We have collected data respectively with three tool configurations.

- $\operatorname{Tbck}()$ represents the one that uses $\operatorname{Tbck}()$ for all time progress evaluation.

- Cascading represents the one with the technique in section 5.

- $A T C C$ represents the one with the technique in section 6.

The performance data is reported in table 1 . The CPU time used, the total memory consumption for the data-structures in state-space representations, and the 
answers of model-checking are reported. As can be seen, our technique in section 5 always performs better than Tbck(). For some benchmark, the performance enhancement is one order of magnitude. This shows that our techniques could be useful in applying TCTL model-checking technology to industrial projects.

The technique in section 6 did not perform as well as we expected. Further investigation revealed that special arrangement for garbage CRD nodes might have slowed down the hash table operations and blown up the memory consumption. In our present implementation, garbage collection cannot be invoked inside the procedures for the technique. In the future, we may gain more performance with an implementation of a more powerful garbage collector.

\section{Concluding remarks}

In this work, we discuss how to improve the performance of an important component algorithm, the time progress evaluation algorithm, for the model-checking of TAs. Techniques in section 6 may worth further investiagtion for better precision in the approximation and more efficient algorithms.

\section{References}

1. R. Alur, C. Courcoubetis, D.L. Dill. Model Checking for Real-Time Systems, IEEE LICS, 1990.

2. R. Alur, C.Courcoubetis, T.A. Henzinger, P.-H. Ho. Hybrid Automata: an Algorithmic Approach to the Specification and Verification of Hybrid Systems. Workshop on Theory of Hybrid Systems, LNCS 736, Springer-Verlag, 1993.

3. R. Alur, D.L. Dill. A Theory of Timed Automata. Theoretical Computer Science, 126:183-235, 1994.

4. J. Bengtsson, K. Larsen, F. Larsson, P. Pettersson, Wang Yi. UPPAAL - a Tool Suite for Automatic Verification of Real-Time Systems. Hybrid Control System Symposium, 1996, LNCS, Springer-Verlag.

5. D.L. Dill. Timing Assumptions and Verification of Finite-state Concurrent Systems. CAV'89, LNCS 407, Springer-Verlag.

6. J.B. Fourier. (reported in:) Analyse des travaux de l'Académie Royale des Sciences pendant l'année 1824, Partie Mathématique, 1827.

7. T.A. Henzinger, X. Nicollin, J. Sifakis, S. Yovine. Symbolic Model Checking for Real-Time Systems, IEEE LICS 1992.

8. F. Wang. Efficient Verification of Timed Automata with BDD-like Data-Structures, STTT (Software Tools for Technology Transfer), Vol. 6, Nr. 1, June 2004, SpringerVerlag; special issue for the 4th VMCAI, Jan. 2003, LNCS 2575, Springer-Verlag.

9. F. Wang. Symbolic Parametric Safety Analysis of Linear Hybrid Systems with BDD-like Data-Structures. IEEE Transactions on Software Engineering, Volume 31, Issue 1 (January 2005), pp. 38-51, IEEE Computer Society. A preliminary version is in proceedings of 16th CAV, 2004, LNCS 3114, Springer-Verlag.

10. F. Wang, G.-D. Huang, F. Yu. TCTL Inevitability Analysis of Dense-Time Systems: From Theory to Engineering. IEEE Transactions on Software Engineering, Vol. 32, Nr. 7, July 2006, IEEE Computer Society.

11. S. Yovine. Kronos: A Verification Tool for Real-Time Systems. International Journal of Software Tools for Technology Transfer, Vol. 1, Nr. 1/2, October 1997. 